\title{
Immune-checkpoint inhibitors for lung cancer patients amid the COVID-19 pandemic: a case report of severe meningoencephalitis after switching to an extended-interval higher flat-dose nivolumab regimen
}

\author{
Anna Pous ${ }^{1 \#}$, Cristina Izquierdo ${ }^{2 \#}$, Marc Cucurull ${ }^{1,3,4}$, Silvia Sánchez ${ }^{1}$, Clara Lezcano ${ }^{5}$, Marta Domenech ${ }^{1,3,4}$, \\ Laia Llobera ${ }^{1}$, Andrea Plaja ${ }^{1}$, Teresa Moran ${ }^{1,3,4,6}$ \\ ${ }^{1}$ Medical Oncology Department, Catalan Institute of Oncology Badalona, Hospital Universitari Germans Trias i Pujol, Barcelona, Spain; ${ }^{2}$ Neurology \\ Department. Hospital Universitari Germans Trias i Pujol, Barcelona, Spain; ${ }^{3}$ Institut Germans Trias i Pujol, Barcelona, Spain; ${ }^{4}$ Badalona Applied \\ Research Group in Oncology, Barcelona, Spain; ${ }^{5}$ Pharmacy Department, Catalan Institute of Oncology Badalona, Hospital Universitari Germans \\ Trias i Pujol, Barcelona, Spain; 'Department of Medicine, Universitat Autònoma de Barcelona (UAB), Campus Can Ruti, Barcelona, Spain \\ \#These authors contributed equally to this work. \\ Correspondence to: Teresa Moran, MD, PhD. Medical Oncology Department, Catalan Institute of Oncology Badalona, Hospital Universitari Germans \\ Trias i Pujol, Barcelona; Spain Institut Germans Trias i Pujol, Barcelona, Spain; Badalona Applied Research Group in Oncology, Barcelona, Spain; \\ Department of Medicine, Universitat Autònoma de Barcelona (UAB), Campus Can Ruti, Barcelona, Spain. Email: mmoran@iconcologia.net.
}

\begin{abstract}
Alternative dosage regimens for some anticancer therapies have been proposed in the midst of the SARS-COV-2 pandemic in order to protect the patients from attending to health care facilities. Flatdosing of several immune-checkpoint inhibitors (ICIs), including nivolumab, have been established. Although generally well tolerated with no new safety signals, new dosages can associate novel individual toxicities. As the use of ICIs is increasing in cancer patients, the present case report is a reminder for clinicians of potential novel toxicities, as well as the need for an interdisciplinary approach for their recognition and treatment. We report the occurrence of a severe neurologic toxicity in a patient with non-small cell lung cancer (NSCLC) who developed should be changed to which occurred after two doses of extended higher interval flat-dose nivolumab despite two years of clinical stability on prior nivolumab regimen. Patient developed fever, language impairment and altered mental status. The work-up tests excluded other potential causes and the most likely diagnosis was meningoencephalitis. Fortunately, with medical treatment, which consisted of high dose steroids, the patient recovered to his baseline situation and symptoms did not recurred, even though nivolumab was resumed. Alternate ICI regimens may have unique immune-related adverse event profiles.
\end{abstract}

Keywords: Extended-interval higher flat-dose; nivolumab; meningoencephalitis; non-small cell lung cancer (NSCLC); case report

Submitted Dec 31, 2020. Accepted for publication Feb 22, 2021.

doi: $10.21037 /$ tlcr-20-1315

View this article at: http://dx.doi.org/10.21037/tlcr-20-1315

\section{Introduction}

SARS-COV-2 infection has had an impact on cancer patient care. Lung cancer patients, were considered a highly vulnerable population (1). With no available guidelines, Oncology Departments had to redesign how to provide adequate patient care (2). At our institution, therapeutic protocols were modified according to the COVID-19 patient load.

Immune-checkpoint inhibitors (ICI) have been proven efficacious in patients with non-small cell lung cancer (NSCLC) (3). The anti-PD-1 nivolumab has demonstrated efficacy in second-line regardless of histology (4). Despite 
the changing landscape in first-line therapy in NSCLC, with more patients accessing ICI at earlier stages, some patients were still receiving second -line nivolumab in our department. Patients were rescheduled to receive an extended-interval higher flat-dose. This strategy consists of both increase the dose and extend the interval between doses for the patients, from once every two weeks (Q2W) to every four weeks $(\mathrm{Q} 4 \mathrm{~W})(5)$. This approach has been considered safe and efficacious (6), although higher flatdose might associate a change in immune-related adverse events (irAE) profile.

However, clinicians need to be aware of potential harms of new dosages. Here, we report the case of a patient who presented a severe neurologic toxicity once nivolumab was given higher flat-dose. The article is in accordance with the CARE reporting checklist (available at http://dx.doi. org/10.21037/tlcr-20-1315).

\section{Case presentation}

A 68-year-old-male with no past medical history except for anxiety and depression treated with clonazepam and venlafaxine, consulted in February 2018, because of chest pain. A CT-Scan revealed a lower left lobe mass with left pleural effusion and left adrenal mass metastases (M1). The bronchoscopy, pleural fluid drainage and EBUS with primary tumor sampling returned non-diagnostic. The left adrenal gland biopsy resulted positive for adenocarcinoma. Molecular testing including EGFR, ALK, ROS1 and $K R A S$ was negative, as well as PD-L1 expression. Thus, the final diagnosis was stage IVA adenocarcinoma of the lung (cT4N0M1b). The patient was started on cisplatin $75 \mathrm{mg} / \mathrm{m}^{2}$ and pemetrexed $500 \mathrm{mg} / \mathrm{m}^{2} / 3$ weeks. He received four cycles from March to May 2018. A new adrenal gland biopsy was performed and testing returned negative for any molecular alteration. PD-L1 expression in this second sample was $60 \%$ (Figure 1).

After four cycles of chemotherapy, the CT-Scan revealed progressive disease (PD) with enlargement of the adrenal $\mathrm{M} 1$, and he was started on nivolumab $3 \mathrm{mg} / \mathrm{kg} / \mathrm{Q} 2 \mathrm{~W}$ (total dose of $240 \mathrm{mg}$ according to weight of $80 \mathrm{~kg}$ ) in June 2018. He received a total of 46 cycles with partial response (PR). He presented grade 1 arthralgias and low fever after nivolumab during the first two months of treatment. Nivolumab higher flat-dosing was proposed in April 2020 amid the SARS-COV-2 pandemic. He received two cycles of nivolumab $480 \mathrm{mg} / \mathrm{Q} 4 \mathrm{~W}$.

Twenty-four hours after the first higher flat-dose administration, the patient presented low fever, slowed mental response, somnolence, arthralgia, asthenia, and hand tremor with a spontaneous complete remission in 10 days. These symptoms were unreported. Ten days after the administration of a second higher flat-dose, he was transferred to the emergency room (ER) after 12-hour of fever syndrome, altered mental status with visual hallucinations, headache, language impairment, gait instability and psychomotor agitation. Concomitant medication at time of admission had remained unchanged for several months and consisted of clonazepam $0.6 \mathrm{mg}$ Q.D., venlafaxine $75 \mathrm{mg}$ Q.D. and an $87 \mu \mathrm{g} / 72 \mathrm{~h}$ transdermal fentanyl patch. No alcohol or other substances use was reported. At the ER, the patient presented temporo-spatial disorientation with mixed aphasia. Cranial nerves were intact, the muscular balance was normal and there was no loss of sensitivity. He presented bilateral upper limb asterixis with no dysmetria and gait instability with a positive Romberg test. The systemic exam was unremarkable.

A brain CT-Scan with contrast ruled out metastases, hemorrhage or acute ischemic stroke. Blood test, urine test and chest-X-ray showed no findings. COVID-19 PCR was negative. No specific biochemistry or urine screen panel to detect alcohol or other drugs was performed. Lumbar puncture (LP) revealed an inflammatory CSF with $7 \mathrm{cell} / \mathrm{mm}^{3}$ (all of them lymphocytes) and $0.76 \mathrm{~g} / \mathrm{L}$ of protein.

A comprehensive microbiological screening, which included bacterial cultures, and Herpes Simplex virus, Herpes-6 virus, Varicella Zoster virus and Epstein Barr virus PCR as well as malignant cells screening in the CSF were negative. VZV CFS IgG and IgM were not tested.

Neither oligoclonal bands, nor onconeuronal or surface antibodies (Abs) were detected in the CSF or in serum. The immunologic panel in CSF and serum included the following: oligoclonal bands, onconeural Ab (anti-Tr (DNER), anti-GAD65, anti-Zic4, anti-titin, anti-SOX1, anti-Recoverin, anti-Hu, anti-Yo, anti-Ri, anti-PNMA2, anti-CV2, anti-Amphiphysin) and surface Ab (antiNDMA-R, anti-AMPAR1/2, anti-DPPX receptor, antiGABA(b)R, anti-LGI1 receptor, anti-CASPR2 receptor).

Main differential diagnosis comprised infectious or ir-meningoencephalitis, and leptomeningeal disease. Consequently, iv. acyclovir and high dose of metilprednisolone $(1 \mathrm{mg} / \mathrm{kg})$ were started empirically. A brain MRI did not show enhancements suggesting brain metastases or leptomeningeal involvement. T2 and FLAIR signal revealed slight periventricular changes attributable 


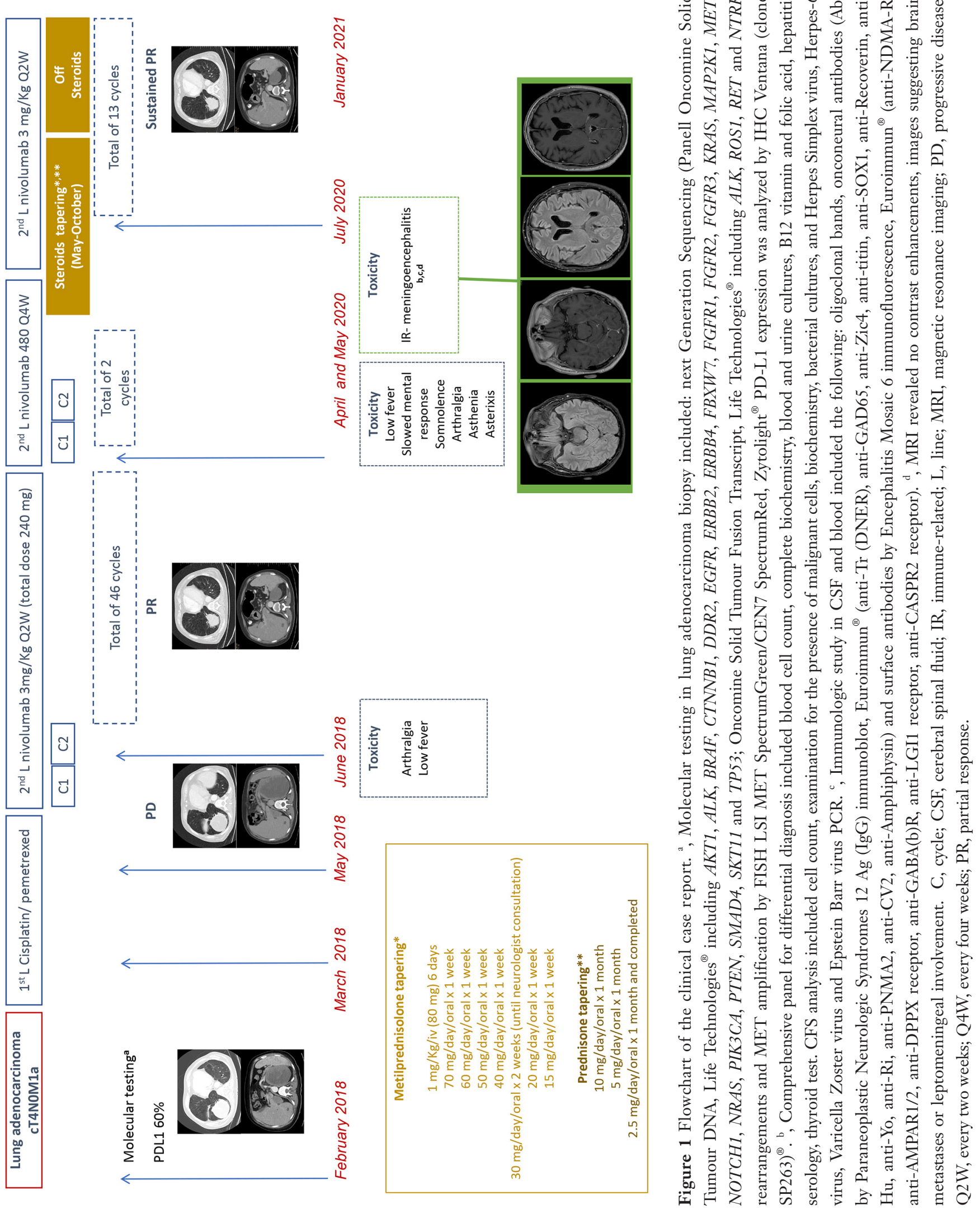


to the involution age-related process. Empirical treatment with acyclovir was stopped after Varicella Zoster PCR and brain MRI results. During the hospital stay, the patient presented a favorable outcome. At discharge, he had mild slowed mental response and disorientation with no language impairment, and he was able to walk without assistance.

Fifteen days after discharge, a new LP showed 4 cell $/ \mathrm{mm}^{3}$, normal levels of glucose and protein, and cytology was negative for malignant cells. In the following weeks, the patient experienced a progressive cognitive recovery.

Patient received $1 \mathrm{mg} / \mathrm{kg}$ iv metilprednisolone (total dose $80 \mathrm{mg} /$ day) while admitted in the hospital. At discharge, tapering of $10 \mathrm{mg} /$ week was recommended under close surveillance. In July 2020, once the patient was on $20 \mathrm{mg} /$ day of metilprednisolone with no symptoms recurrence, nivolumab $3 \mathrm{mg} / \mathrm{kg} / \mathrm{Q} 2$ was resumed, since patient was willing to continue therapy which provided an adequate tumor control and despite the irAE occurrence; and the steroids were tapered to $15 \mathrm{mg} /$ day for a week and then to $10 \mathrm{mg} / \mathrm{day}$, at this point with prednisone to ease the tapering. From then on, in mid-July, steroids were tapered for longer intervals (to $10 \mathrm{mg} /$ day a month) and then halved to 5 and $2.5 \mathrm{mg} /$ day a month, respectively. Without concerning symptoms recurrence, steroids were completed in October 2020. He received 7 cycles of nivolumab while tapering the steroids (from July to October 2020) and 6 cycles off steroids (from November 2020 to January 2021). Nivolumab has been continued without new neurological toxicity and a sustained PR.

All procedures performed in studies involving human participants were in accordance with the ethical standards of the institutional and/or national research committee(s) and with the Helsinki Declaration (as revised in 2013). The patient gave his written informed consent for his personal and clinical details along with any identifying images to be published in this study.

\section{Discussion}

ICI are approved in different clinical settings in NSCLC, with proven efficacy and a favorable irAE profile. ICI are associated with a novel spectrum of toxicities; most irAE are mild or moderate (7).

Although uncommon, neurological irAE might be severe with permanent sequels and potentially life-threating $(8,9)$. Because of a lack of prospective studies focused on assessing ICI-related neurotoxicity, its incidence is probably underestimated. Approximately, $12 \%$ of patients treated with ICI will develop neurotoxicity (10), being less frequent with anti-PD-1/PD-L1 $(6.1 \%)$ or anti-CTLA (3.8\%) alone. Importantly, less than $1 \%$ will present severe complications (10). Clinical presentation of neurological irAE are varied and may involve peripheral or central nervous system (CNS). Peripheral nervous system complications are more frequent (11). They are rarely related to the presence of Abs and more frequent in patients with melanoma treated with anti-CTLA4 and display a better prognosis (11).

The diagnosis of CNS ir-neurotoxicity remains challenging. Three main clinical phenotypes with distinct immunological background have been described (11). Meningoencephalitis and limbic encephalitis (LE) are the most frequent phenotypes, but differ in clinical, radiological and CSF features (Table 1) (11). When well-characterized anti-neural-Abs can be identified, these cases can be considered as paraneoplastic neurological syndromes (PNS). Unlike non-ICI-PNS, post-ICI-PNS are diagnosed in advanced phases of the disease, even in cases with known brain metastases (12). Post-ICI-PNS onset has been related to a latent immune response directed against CNS and mediated by neural antigens expressed by tumor cells (13). Detection of anti-neural-Abs in asymptomatic patients who developed post-ICI-PNS supports this hypothesis (14).

Our patient was diagnosed with ir-meningoencephalitis after ruling out $\mathrm{CNS}$ infection, and leptomeningeal or brain involvement. Onconeural-Abs were not detected, neither in serum nor in CSF. They were analyzed though commercial immunodots without a subsequent confirmation by Cellbased Assays or immunohistochemistry. Furthermore, detection of GFAP antibody, which had been identified in ir-meningoencephalitis, was not included in the commercial kit (11).

A striking point is that our patient had been treated with nivolumab for 22 months with no signs or symptoms of neurotoxicity, which occurred when patient was transitioned to higher flat-dose. Despite neurotoxicity is more often detected within the first months, it may appear at any given time during the course of treatment. Assessment of nivolumab exposure and clinical safety of $480 \mathrm{mg} / \mathrm{Q} 4 \mathrm{~W}$ higher flat-dosing schedule demonstrated a similar timeaveraged concentration compared with $3 \mathrm{mg} / \mathrm{kg}$ Q2W when PK was simulated in 3,871 patients across multiple tumor types, including NSCLC (5). Regarding irAE, no new safety signals were identified in a pooled safety data from these patients and from 61 patients who transitioned from $3 \mathrm{mg} / \mathrm{kg} / \mathrm{Q} 2 \mathrm{~W}$ to $480 \mathrm{mg} / \mathrm{Q} 4 \mathrm{~W}$. Moreover, a PK modelbased evaluation demonstrated similar PK profile with 
Table 1 Differential diagnosis of immune-related adverse events involving central nervous system (11)

\begin{tabular}{lll}
\hline Variable & Limbic encephalitis & Meningoencephalitis \\
\hline Symptoms & Altered mental status & Fever \\
& Anterograde memory disturbances & Headache \\
& Seizures & Language impairment \\
& Psychiatric disorders & Altered mental status \\
& & Potential peripheral nervous system involvement \\
Brain MRI & Mesial temporal lobe hypersignal or normal & Leptomeningeal, perivascular enhancement or normal \\
CFS findings & Inflammatory findings+ & Inflammatory findings++ \\
& anti-neural Abs++ (Ma2) & anti-neural Abs + (GFAP)
\end{tabular}

*Inflammatory CSF: pleocytosis, presence of oligoclonal bands and/or proteinorrachia. Ab, antibodies; GFAP, Glial Fibrillary Acidic Protein.

nivolumab $480 \mathrm{mg} / \mathrm{Q} 4 \mathrm{~W}$ vs. $3 \mathrm{mg} / \mathrm{kg} / \mathrm{Q} 2 \mathrm{~W}$, with a comparable prediction for both response and irAE irrespective of the nivolumab dose and across tumor types (6). Checkmate 384 demonstrated no differences between nivolumab $480 \mathrm{mg} / \mathrm{Q} 4 \mathrm{~W}$ vs. $240 \mathrm{mg} / \mathrm{Q} 2 \mathrm{~W}$ in terms of both post randomization progression-free survival and median duration of treatment, as well as in irAE rate in NSCLC patients previously exposed to nivolumab for a period of $\leq 12$ months (15). Severe irAE occurred in the range of $5 \%$ or less. Thus, higher flat-dose of nivolumab represents a convenient option to reduce the attendance to health care facilities, especially for those patients whose body weight is around $80 \mathrm{~kg}$. However, another group have reported a case of bullous pemphigoid associated with extended-interval flat $480 \mathrm{mg}$ nivolumab dose regimen in a patient with a metastatic renal carcinoma who had been previously treated with $240 \mathrm{mg}$ Q2W for 2 years without safety concerns (16). While our case report and others suggest these extendedinterval higher flat-dose regimens may increase or alter the propensity for ICI irAEs, clinical trial data does suggest these regimens have similar efficacy and safety profiles and are reasonable options for patients. However, when switching between ICI dosing regimens providers and patients need to have increased vigilance for unique irAE risks when compared to traditional dosing.

Eighteen patients were transitioned from nivolumab $3 \mathrm{mg} / \mathrm{kg} / \mathrm{Q} 2 \mathrm{~W}$ to $480 \mathrm{mg} / \mathrm{Q} 4 \mathrm{~W}$ amid the COVID-19 pandemics at our institution. Nine, seven and two patients received three, two and one cycles of nivolumab Q4W between March and May 2020, respectively. Median cycles of prior nivolumab were 42 [1-110]. In June 2020, fifteen patients continued with nivolumab Q2W with a median of 5 additional cycles [0-7]. Three patients completed nivolumab during this period (2 due to PD, 1 other reason). Four patients developed a more prolonged, but grade 1, asthenia during the Q4W dosage. No additional toxicities were reported. In June 2020, 3 patients continued with nivolumab Q2W while 12 patients were definitively transitioned to Q4W, with a median of 8 additional cycles [1-11]. Subsequent evaluations have demonstrated sustained responses in all patients.

When ir-neurotoxicity is suspected, the therapeutic approach includes discontinuation of ICI until the nature of irAE is defined, with multidisciplinary management and supportive treatment (8). Whereas in grade 1 or 2 toxicity, oral prednisolone $(0.5 \mathrm{mg} / \mathrm{kg})$ usually allows symptom control, in grade 3 or 4 toxicity high-dose steroid therapy with oral prednisolone $(1-2 \mathrm{mg} / \mathrm{kg}$ ) or iv. equivalent should be proposed. Additionally, plasmapheresis or iv. immunoglobulin may be required when there is an incomplete response or clinical worsening.

There is limited data on the safety of rechallenging with ICI after ir-neurotoxicity (17). Interestingly, a large retrospective study analyzed 24,079 cases with at least one ICI-related AE (18). Nearly $30 \%$ of patients presented a recurrence of the initial irAE when ICI was resumed. Three out of 19 patients presented recurrence of neurotoxicity. Guidelines recommend permanent discontinuation of ICI specially in patients who have presented a grade 4 toxicity (8). Nevertheless, resuming ICI therapy concomitantly with immunosuppressive therapy may be considered for selected patients.

Since our patient had the disease under control for more than 2 years, and neurological irAE occurred in the setting of a higher flat-dose with a good response to corticosteroids, nivolumab was resumed. Patient was carefully monitored 
and treatment with corticosteroids was maintained for 5 months. After steroids discontinuation patient is still asymptomatic and neurological examination is normal. In our case, we speculate that this neurological irAE was related to the higher flat-dose of nivolumab. Having resumed the initial dose of ICI with corticosteroid may have played a role in the absence of neurotoxicity recurrence.

\section{Conclusions}

Altering ICI schedules including to an extended-interval higher flat-dose regimen may alter the risk of severe irAEs. Once the irAE is treated, resumption of the ICI at previously well-tolerated dose initially with low doses of corticosteroids could be an efficacious and safe option in selected patients.

\section{Acknowledgments}

We thank the patient who signed an IRB approved informed consent form that allowed this case report publication.

Funding: None.

\section{Footnote}

Reporting Checklist: The authors have completed the CARE reporting checklist. Available at http://dx.doi.org/10.21037/ tlcr-20-1315

Peer Review File: Available at http://dx.doi.org/10.21037/ tlcr-20-1315

Conflicts of Interest: All authors have completed the ICMJE uniform disclosure form (available at http://dx.doi. org/10.21037/tlcr-20-1315). The authors declare no conflict of interest related to the current work. APous has received personal fees from Roche, MSD and Rovi. MC has received personal fees from Roche, Pharmamar and Merck. TM has received honoraria for consultancy and lectures from Astra Zeneca Bristol Myers Squibb and MSD and personal fees from Roche. APlaja has received personal fees from MSD, Roche y Angelini. MD has received personal fees from Roche, Astra Zeneca and Bristol Myers Squibb. The other authors have no conflicts of interest to declare.

Ethical Statement: The authors are accountable for all aspects of the work in ensuring that questions related to the accuracy or integrity of any part of the work are appropriately investigated and resolved. All procedures performed in studies involving human participants were in accordance with the ethical standards of the institutional and/or national research committee(s) and with the Helsinki Declaration (as revised in 2013). The patient gave his written informed consent for his personal and clinical details along with any identifying images to be published in this study.

Open Access Statement: This is an Open Access article distributed in accordance with the Creative Commons Attribution-NonCommercial-NoDerivs 4.0 International License (CC BY-NC-ND 4.0), which permits the noncommercial replication and distribution of the article with the strict proviso that no changes or edits are made and the original work is properly cited (including links to both the formal publication through the relevant DOI and the license). See: https://creativecommons.org/licenses/by-nc-nd/4.0/.

\section{References}

1. Liang W, Guan W, Chen R, et al. Cancer patients in SARS-CoV-2 infection: a nationwide analysis in China. Lancet Oncol 2020;21:335-7.

2. Lambertini $M$, Toss A, Passaro A, et al. Cancer care during the spread of coronavirus disease 2019 (COVID-19) in Italy: young oncologists' perspective. ESMO Open 2020;5:e00759.

3. Clinical N, Guidelines P, Guidelines N. NCCN Clinical Practice Guidelines in Oncology. Non-Small Cell Lung. Version 8.2020. September 15, 2020.

4. Horn L, Spigel DR, Vokes EE, et al. Nivolumab Versus Docetaxel in Previously Treated Patients With Advanced Non-Small-Cell Lung Cancer: Two-Year Outcomes From Two Randomized, Open-Label, Phase III Trials (CheckMate 017 and CheckMate 057). J Clin Oncol 2017;35:3924-33.

5. Long GV, Tykodi SS, Schneider JG, et al. Assessment of nivolumab exposure and clinical safety of $480 \mathrm{mg}$ every 4 weeks flat-dosing schedule in patients with cancer. Ann Oncol 2018;29:2208-13.

6. Zhao X, Shen J, Ivaturi V, et al. Model-based evaluation of the efficacy and safety of nivolumab once every 4 weeks across multiple tumor types. Ann Oncol 2020;31:302-9.

7. Postow MA, Sidlow R, Hellmann MD. Immune-Related Adverse Events Associated with Immune Checkpoint Blockade. N Engl J Med 2018;378:158-68.

8. Brahmer JR, Lacchetti C, Schneider BJ, et al. Management 
of immune-related adverse events in patients treated with immune checkpoint inhibitor therapy: American society of clinical oncology clinical practice guideline. J Clin Oncol 2018;36:1714-68.

9. Champiat S, Lambotte O, Barreau E, et al. Management of immune checkpoint blockade dysimmune toxicities: a collaborative position paper. Ann Oncol 2016;27:559-74.

10. Cuzzubbo S, Javeri F, Tissier M, et al. Neurological adverse events associated with immune checkpoint inhibitors: Review of the literature. Eur J Cancer 2017;73:1-8.

11. Vogrig A, Muñiz-Castrillo S, Joubert B, et al. Central nervous system complications associated with immune checkpoint inhibitors. J Neurol Neurosurg Psychiatr 2020;91:772-8.

12. Graus F, Dalmau J. Paraneoplastic neurological syndromes in the era of immune-checkpoint inhibitors. Nat Rev Clin Oncol 2019;16:535-48.

13. Vogrig A, Muñiz-Castrillo S, Desestret V, Joubert B, Honnorat J. Pathophysiology of paraneoplastic and autoimmune encephalitis: genes, infections, and checkpoint inhibitors. Ther Adv Neurol Disord

Cite this article as: Pous A, Izquierdo C, Cucurull M, Sánchez S, Lezcano C, Domenech M, Llobera L, Plaja A, Moran T. Immune-checkpoint inhibitors for lung cancer patients amid the COVID-19 pandemic: a case report of severe meningoencephalitis after switching to an extended-interval higher flat-dose nivolumab regimen. Transl Lung Cancer Res 2021;10(4):1917-1923. doi: 10.21037/tlcr-20-1315
2020;13:175628642093279.

14. Vogrig A, Fouret M, Joubert B, et al. Increased frequency of anti-Ma2 encephalitis associated with immune checkpoint inhibitors. Neurol - Neuroimmunol Neuroinflammation 2019;6:e604.

15. Garon EB, Reinmuth N, Falchero L, et al. CheckMate 384: Phase IIIb/IV trial of nivolumab (nivo) $480 \mathrm{mg}$ Q4W versus $240 \mathrm{mg}$ Q2W after $\leq 12$ months of nivo in previously treated advanced NSCLC. J Clin Oncol 2019;37:abstr 100.

16. Palla AR, Smith E, Doll D. Bullous pemphigoid associated with the 480-mg nivolumab dose in a patient with metastatic renal cell carcinoma. Immunotherapy 2019;11:1187-92.

17. Cuzzubbo S, Tetu P, Guegan S, et al. Reintroduction of immune-checkpoint inhibitors after immunerelated meningitis: A case series of melanoma patients. J Immunother Cancer 2020;8:1-5.

18. Dolladille C, Ederhy S, Sassier M, et al. Immune Checkpoint Inhibitor Rechallenge After Immune-Related Adverse Events in Patients With Cancer. JAMA Oncol 2020;6:865. 